\title{
An Interaction Support Processor to Promote Individual and Systemic Benefits
}

\author{
Dirk Helbing
}

\begin{abstract}
Individual choices, if not sufficiently well coordinated, can lead to bad outcomes, such as systemic instabilities or failures, or "tragedies of the commons." It is, therefore, proposed to use digital assistants to support favorable interactions and avoid undesirable ones. The invention discussed here describes ways to perform these tasks in a decentralized way that also protects sensitive information. Such digital assistants offer better solutions based on local empowerment and coordination rather than on large-scale surveillance and control. In particular, it is suggested to introduce a multi-dimensional value exchange based on multiple new currencies that are linked to reputation values or sensor measurements, which may use the Internet of Things. This novel approach expands the solution space such that new opportunities for favorable interactions arise, which benefits the system and its components. Often, similar results would not be achievable with classical optimization approaches and conventional, one-dimensional value exchange only.
\end{abstract}

One day, in late 2012, I decided to write a patent application. It was not for the first time. I had already successfully patented the idea for a self-organized traffic control system together with a colleague. ${ }^{1}$

The new patent application was for an "Interaction Support Processor."2 It described the concept of digital assistants that would not only lead to better individual decisions, but—above all-they would better coordinate people's decisions

This Appendix is a slightly adapted version of the preprint "Interaction Support Processor-and Why the Patenting System Is Broken", available at https://www.researchgate.net/publication/342 040513.

D. Helbing ( $\bowtie)$

ETH Zurich, Computational Social Science, Stampfenbachstrasse 48, 8092 Zurich, Switzerland e-mail: dhelbing@ethz.ch

${ }^{1}$ D. Helbing and S. Lämmer, Method for coordination of competing processes or for control of the transport of mobile units within a network https://patents.google.com/patent/US8103434B2/en.

${ }^{2}$ Interaction support processor https://patents.google.com/patent/US20160350685A1/en.

(C) The Author(s) 2021

M. M. Dapp et al. (eds.), Finance 4.0-Towards a Socio-Ecological Finance System, SpringerBriefs in Applied Sciences and Technology,

https://doi.org/10.1007/978-3-030-71400-0_5 
so that systemic instabilities and conflicts would be avoided..$^{3}$ The invention was about generating individual advantages, but not at the expense of others. Rather, everyone should benefit!

The patent application proposed a "social mirror", ${ }^{4}$ which would be a digital representation of how certain decisions would affect the environment and others. My goal was to achieve better decisions through "greater awareness," and to make people want to "behave in a more beautiful way"-quite similar to how a mirror makes them want to "look more beautiful."

The patent application also explained how people could be protected from adverse decisions - by a "social protector". In addition, it described, how they could be made aware of favorable opportunities that they would otherwise overlook, namely, through a further kind of digital assistant: a "social guide". This would also ensure that, as far as possible, "win-lose" situations would be turned into "win-win" situations (by means of compensation payments), so that all parties would benefit from the interaction. For this purpose, the patent application proposed "social money"- new types of money that could, for example, depend on reputation or measurements (see Fig. 1). ${ }^{5}$

The ultimate goal, which should be achieved by the invention, was a world without manipulation, exploitation, and coercion. A world where people and the environment would benefit from digitally assisted, considerate behavior, and from actions that would benefit us all. All this would happen in a way that would use digital technologies for personal empowerment, while protecting our privacy.

The patent, I hoped, would offer a way into a positive digital future, and a way out of the current dystopia. With the patent, I wanted to create opportunities for companies and people, who were engaged for a better future, while there would be obstacles for those, who just cared about profit.

Let me make a bit clearer how the invention would work, so that, in the future, many would benefit, not just a few, as it had been the case in the past. To do this, I will shortly summarize the idea of "social money".

Imagine that we would have measurement methods that measure $\mathrm{CO}_{2}$, noise, or toxins, or also good things such as resources of various kinds: glass, plastic, metalsor health, knowledge, social, or cultural achievements. Moreover, imagine we do not give them a price in Dollars or Euros, but we measure them in different currencies

\footnotetext{
${ }^{3}$ This idea was somewhat similar to how our traffic assistance systems worked, see e.g. A. Kesting, M. Treiber, M. Schöhof, and D. Helbing (2008) Adaptive cruise control design for active congestion avoidance, Transportation Research C 16(6), 668-683, https://www.sciencedirect.com/science/art icle/pii/S0968090X08000028; S. Lämmer and D. Helbing (2008) Self-control of traffic lights and vehicle flows in urban road networks, J. Stat. Mech., P04019, https://iopscience.iop.org/article/ 10.1088/1742-5468/2008/04/P04019/meta; D. Helbing (2013) Economics 2.0: The natural step towards a self-regulating, participatory market society. Evolutionary and Institutional Economics Review 10, 3-41, https://link.springer.com/article/10.14441/eier.D2013002.

${ }^{4}$ Social mirror: More success through awareness and coordination, https://link.springer.com/cha pter/10.1007/978-3-319-90869-4_17.

${ }^{5}$ Note that the concept of "social money" is somewhat similar to the concept of "qualified money" introduced before.
} 


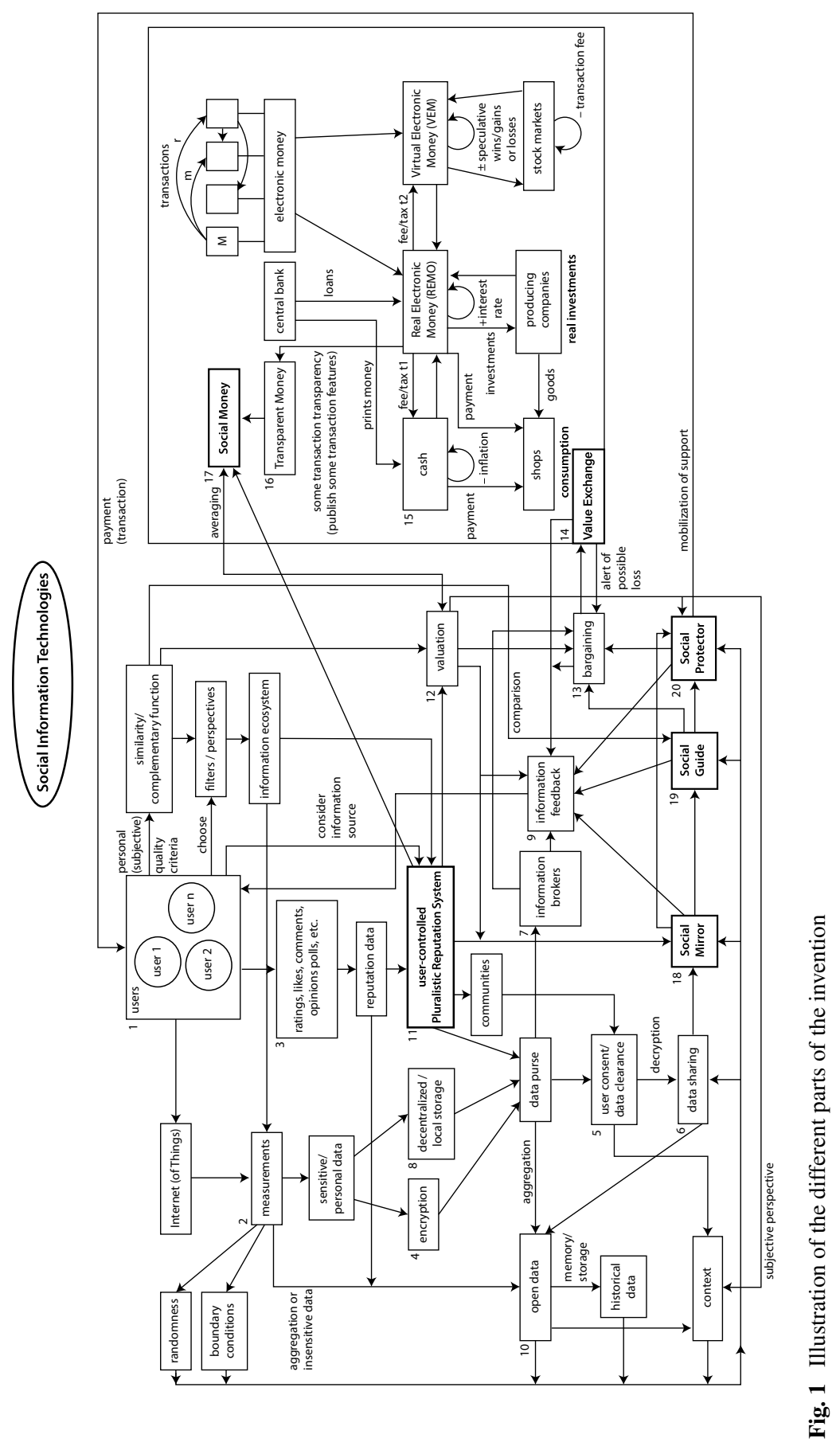




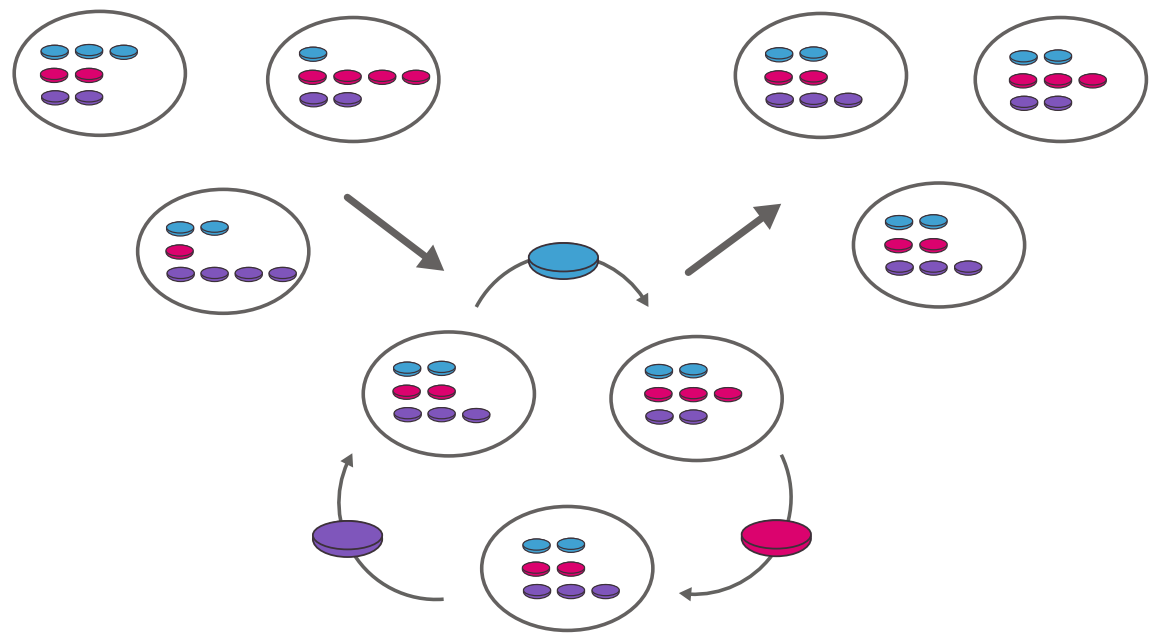

Fig. 2 Illustration of multi-dimensional value exchange, here, a payment process involving and benefitting multiple interaction partners

that cannot be easily exchanged for each other-only at a considerable fee. So, in a sense, we would manage different kinds of values with separate accounts.

Instead of a one-dimensional monetary system, where everything can be converted in an almost frictionless way into Dollars or Euros - and where everything can be bought with one kind of money - a multi-dimensional monetary system would be created (see Fig. 2). ${ }^{6}$ This system would no longer be primarily about profit maximization. Social values would matter, too, ecological values as well. And one could also consider cultural values.

Everyone could contribute to the system by various kinds of value creation-in whatever way it suits their talents and interests. When we buy goods or services, we would pay with a mix of currencies to compensate for their social, environmental, and cultural values. So, we would commit not only to profit maximization, but also to achieving social, environmental, and cultural goals, in order to earn the currencies we need. Otherwise, we would have to pay a considerable exchange fee to get the currencies we lack. With an adequate mobile phone app, however, all payment processes would be very easy.

A multi-dimensional monetary system as described above would create a multidimensional real-time feedback system. This would be much more suitable for the control-or even self-organization-of complex systems than the basically onedimensional monetary system of today. ${ }^{7}$ Instead of the current economic organization, which wastes a lot of resources, a system would emerge that would work

\footnotetext{
${ }^{6} \mathrm{cf}$. https://www.youtube.com/watch?v=PJGZpV4PUwY.

${ }^{7}$ Qualified Money_A better financial system for the future, https://papers.ssrn.com/sol3/papers. cfm?abstract_id $=2526022$, published in this book.
} 
similar to nature: a resource-saving circular economy. ${ }^{8}$ It would be a new system made possible by a new approach: through multi-dimensional co-evolution rather than one-dimensional optimization: through coordination instead of control. This would create a new kind of economy that would boost a (more) sustainable world and might, thereby, be able to save millions of lives.

With such a multi-dimensional system, the economy could be much better steered in directions that serve the environment and humanity. Several goals could be pursued simultaneously - not just profit maximization. The world would continue to improve through a co-evolutionary process. And we could all participate in it!

\section{Some Background}

The invention is focused on interactions among smart system components capable of sensing, information processing, valuation, and information exchange, which could be smart devices such as AI systems, intelligent machines, bots, or robots, or also people using smart devices (i.e., networks of system components, where the components comprise people and technology). For example, the interaction support processor could be a particular, novel kind of personal digital assistant. Detailed specifications are made in the main body of the patent. ${ }^{9}$

Sensing (sensor measurements) play(s) a role

- to determine and valuate the local context of the prospective actions and interactions, and of alternative actions and interactions (where possible interventions or possible value exchanges have been added), ${ }^{10}$

- for the measurement of the actions and interactions in the system and their effects (e.g., for a data-driven valuation),

- for a data-driven modeling and simulation of the action and interaction effects,

- for sensor-measurement-based kinds of value exchanges.

Information processing plays a role for data management, for the determination of possible interventions and possible value exchanges, and for the valuation of actions and interactions with and without these.

Valuation is needed to compare a scenario given by prospective actions and interactions with scenarios given by these prospective actions and interactions when possible interventions and possible value exchanges are added to them.

Information exchange is needed to communicate between different system components, particularly in a distributed, privacy-protecting implementation.

\footnotetext{
${ }^{8}$ The FIN4 Project: Towards a Socio-Ecological Finance System, by Dirk Helbing, https://www. youtube.com/watch? $\mathrm{v}=$ XnemIMW7e3c.

${ }^{9}$ See [0024]f, [0047], [0117] in https://patents.google.com/patent/US20160350685A1/en.

${ }^{10}$ Throughout the main body of the patent, many ways of determining context by means of modern digital technologies have been described, see https://patentscope.wipo.int/search/en/det ail.jsf?docId=WO2015118455.
} 
In contrast to what we have today, we are talking here mainly about a multidimensional value exchange system. ${ }^{11}$ This is important as it increases the optimization space and, therefore, the set of possible solutions, and hence allows for better solutions than without the consideration of this multi-dimensional value exchange system. It creates additional possibilities to improve the system as compared to today's scenario analysis techniques or current monetary compensation schemes in our economy. The proposed multi-dimensionality of value exchange is the main reason why the invention allows to find solutions that benefit all system components - in contrast to the systems known today.

The invention focuses on interventions that consider a plurality of value exchanges. This implies that not only feedback effects are being considered, as this is being done by applied "scenario analysis," but that additionally value exchanges are explored. The consideration of multi-dimensional digitally based value exchanges ${ }^{12}$ is one of the aspects, which sets the invention apart from the state-of-the art in technological, social or economic systems at the time this patent application was submitted.

What the invention proposes is very different from what is being done and discussed today, where each thing, e.g., $\mathrm{CO}_{2}$, glass bottles for recycling, poisons, or any other kind of externalities is given a certain value or price in Dollars, say, and where there is quite frictionless exchange between different kinds of currencies or assets, which makes today's money-based feedback system effectively onedimensional (i.e., there is one overall price, which supports utilitarian approaches). In contrast, the system proposed here is designed in such a way that it is a multidimensional real-time feedback system in an action space that has been extended by the possibility to exchange multiple kinds of values. Moreover, the invention specifies novel kinds of (monetary) values, which are defined on the basis of measurements or reputation values, for example.

Further aspects can be illustrated for the case, where a system component's valuation of prospective actions and interactions is done via a goal function $G$. In classical optimization, a goal function $G(x)$ is optimized as a function of some variables $x$ (where $x$, accordingly, may represent a vector). As $G$ is a one-dimensional quantity, one can always say whether a solution $G\left(x_{1}\right)$ is better $(>)$, worse $(<)$, or equal $(=)$ in quality. Otherwise (i.e., for a multi-dimensional goal function), the classical method of optimization does not work.

If you have two or more goal functions $G_{1}, G_{2} \ldots$, this kind of $>,<,=$ comparison cannot be done. As one changes $x$, one goal may be better achieved and the other one may take on a worse value [i.e., $G_{1}\left(x_{1}\right)>G_{1}(x)$, while $G_{2}\left(x_{1}\right)<G_{2}(x)$ ]. The invention describes what to do in order to achieve solutions where two or more goal functions are simultaneously improved. Such a solution often does not exist with one kind of value exchange. It requires a multi-dimensional value exchange system.

\footnotetext{
${ }^{11}$ See [0062], [0146]ff, [0156]ff in https://patents.google.com/patent/US20160350685A1/en.

${ }^{12}$ See [0062], [0146]ff, [0156]ff in https://patents.google.com/patent/US20160350685A1/en.
} 
As this content has not been published anywhere else, below I will provide the revised claim set which had finally been submitted for approval in the USA. ${ }^{13}$

\section{Appendix}

\section{Claims $^{14}$}

1. A computer-implemented method, comprising:

under the control of one or more computer systems configured with executable instructions,

maintaining system component data of a plurality of system components in a system component data structure;

maintaining transaction data of a plurality of transactions in a transaction data structure, wherein a transaction in the plurality of transactions is a record of an interaction between two or more system components of the plurality of system components;

evaluating components of a prospective transaction among a set of prospective system components, wherein the components include whether each system component of the set of prospective system components has an initial positive valuation of the prospective transaction thereby providing a favorable interaction transaction as to such system components, and which, if any system component of the set of prospective system components has an initial negative valuation of the prospective transaction thereby providing an unfavorable interaction transaction as to such system components;

determining, based on a computer analysis of the components of the prospective transaction and a rules data structure that defines value exchanges and feedback effects, whether a prospective value exchange or a prospective feedback effect, when added to the prospective transaction, results in an unfavorable interaction transaction as to a particular system

\footnotetext{
${ }^{13}$ There is only this preprint https://www.researchgate.net/publication/342040513, where one can also find the claims I have drafted myself. By the way, my latest claim set starts out with a more technical specification, as it was always intended: A computer-implemented method, comprising a network of system components capable of sensing, information processing, valuation, and information exchange under the control of one or more computer systems configured with executable instructions...

${ }^{14}$ In the meantime, the title of the invention had been changed to "Computer-Based Interactions in Techno-Socio-Economic-Environmental Support Systems with Technical, Social, Economic, and/or Environmental Transaction Management and Processing". Note that it has been stated that these claims would not be patentable in the USA and, hence, the patent application has been abandoned over there. The positive implication of this is that (if I understand patent law correctly) everybody should now be able to use the ideas presented here in the USA and many other countries for free. Note, however, that the application is still pending in some countries.
} 
component being converted into a favorable interaction transaction as to that particular system component;

determining, based on the computer analysis of the components of the prospective transaction and the rules data structure, whether a condition is present in which (1) a proposed value exchange and (2) a proposed feedback effect are present in the rules data structure that, when added to the prospective transaction, results in the prospective transaction being converted into a favorable interaction transaction as to each prospective system component using that prospective transaction; and

outputting, based on whether the condition is present, terms of a modified transaction to each prospective system component, wherein the modified transaction is the prospective transaction modified by the proposed value exchange and the proposed feedback effect.

2. The computer-implemented method of claim 1, wherein the prospective transaction is characterized as to each prospective system component of the prospective transaction as being one of: (1) a win-win situation, (2) a good win-lose situation, (3) a bad win-lose situation, and (4) a lose-lose situation.

3. The computer-implemented method of claim 1 , wherein the proposed value exchange or the proposed feedback effect are based on computations done using data provided by a third-party broker.

4. The computer-implemented method of claim 1, wherein the proposed value exchange and the proposed feedback effect further include determining expected behaviors and social norms according to averages of social behaviors over actual measured behaviors.

5-7. (Canceled)

8. A computer-implemented method, comprising:

under the control of one or more computer systems configured with executable instructions,

maintaining system component data of a plurality of system components in a system component data structure;

maintaining transaction data of a plurality of transactions in a transaction data structure, wherein a transaction in the plurality of transactions is a record of an interaction between two or more system components of the plurality of system components;

evaluating components of a prospective transaction among a set of prospective system components, wherein the components include relative valuations of the set of prospective system components participating in the prospective transaction;

aligning value changes of a system and at least one component of the prospective transaction according to a respective valuation of interactions or potential interactions;

determining, based on computer analysis of the components of the prospective transaction and a rules data structure that defines value exchanges and feedback effects, whether a prospective value exchange or a prospective 
feedback effect, when added to the prospective transaction, results in an unfavorable interaction transaction as to a particular system component being converted into a favorable interaction transaction as to that particular system component; and

flagging the prospective transaction as being one of a favorable transaction, an unfavorable transaction, or a semi-favorable transaction, wherein a semi-favorable transaction is defined as a transaction that is convertible to a favorable transaction via a bargaining and value exchange, wherein an unfavorable transaction is defined as a transaction wherein at least one system component of the set of prospective system components has a negative valuation of the prospective transaction, and a favorable transaction is defined as a transaction wherein each system component of the set of prospective system components has a positive valuation of the prospective transaction.

9. (Cancelled)

10. The computer-implemented method of claim 8, wherein the prospective transaction comprises sensitive data and the sensitive data is managed by a third-party broker computer system such that it is not available to each of the plurality of system components.

11. The computer-implemented method of claim 8, further comprising determining reputation values and recommendations according to reputation filters, the reputation filters being personally configurable and shared by system components.

12-15. (Cancelled)

16. The computer-implemented method of claim 8, further comprising: Operating a reputation-based online information filtering system to:

(a) accept a set of ratings, the set of ratings being ratings of online information objects obtained from one or more of the system components;

(b) determine a set of reputation weights, the set of reputation weights being weights of users of the one or more of the system components;

(c) store, into a rating database, data representing the set of ratings and the set of reputation weights;

(d) generate a personal information filter data structure of an information filter derived from the data representing the set of ratings and the set of reputation weights; and

(e) provide viewing user access to the personal information filter data structure, thereby allowing a viewing user to filter online information according to a perspective defined by the set of ratings and the set of reputation weights,

wherein a rating of the set of ratings is a numerical value provided by a rating user for a particular time, weighted by one or more relevance weights and a reputation weight of the rating user and updated over time, rating a specified online information object, 
wherein relevance weights for a posted rating are based on a posting manner in which the posted rating was posted, with a higher relevance weight given to a posting manner that provides greater information about the rating user, wherein online information is presentable to the viewing user filtered according to the personal information filter data structure when the viewing user selects to filter according to the personal information filter data structure, and

wherein a set of reputation weights of the rating user are a function of a manner in which the rating user has previously rated other online information objects.

17. The computer-implemented method of claim 16, wherein the information filter is derived from ratings of a plurality of rating system components.

18. The computer-implemented method of claim 16, wherein the personal information filter data structure is in a form sharable among system components, thereby forming socially sharable information filters, the socially sharable information filters being personally configurable by a receiving system component or automatically configurable according to a context.

19. The computer-implemented method of claim 16, further comprising a value exchange system for system components of the value exchange system to use to transfer value, and wherein value transfers to the rating user are, at least in part, a function of a reputation weight of that rating user.

20. The computer-implemented method of claim 16, further comprising a feedback system, wherein the reputation weight of the rating user varies according to an alignment of the rating user with a set of social media expectations.

21. The computer-implemented method of claim 16, wherein the one or more relevance weights comprise one or more of:

(a) a time span between a rating time when the rating user rated a particular online information object and when that rating is used in a filter,

(b) the posting manner in which the rating user posted the rating, the posting manner being one of anonymously, pseudo-anonymously, or personalized,

(c) a frequency with which the rating user posts ratings,

(d) a number of ratings the rating user posted, and

(e) a quality with which the rating user classified the particular online information object.

22. The reputation-based online information filtering system of claim 21, wherein a first rating posted anonymously is given a first relevance weight, a second rating posted pseudo-anonymously is given a second relevance weight, and a personalized rating identifying the rating user is given a third relevance weight, wherein the first relevance weight is lower than the 
second relevance weight, and the second relevance weight is lower than the third relevance weight.

23. The reputation-based online information filtering system of claim 21, wherein the quality is a function of whether a class, selected from among advertisement, opinion, or fact, into which the rating user classified the particular online information object is a class consistent with a class selected by other system components for the particular online information object.

24. The reputation-based online information filtering system of claim 21, further comprising a filter interaction system, wherein personal information filter data structures are exchanged among system components of the filter interaction system, at least one exchange of which includes a corresponding transfer of value between system components.

25. The reputation-based online information filtering system of claim 16, wherein the rating, by the rating user, of a particular online information object, is weighted by a number of ratings previously made by the rating user, wherein the rating is down-weighted when the number of ratings previously made by the rating user is high or frequent, and wherein the rating is up-weighted when the number of ratings previously made by the rating user is low or infrequent.

26. The reputation-based online information filtering system of claim 16, wherein a rating of the specified online information object over a plurality of rating users is determined according to an equation:

$$
r_{j}(t)=\frac{\sum_{i} r_{i j}\left(t_{i j}\right) f_{i j}\left(t_{i j}\right) w_{i}\left(t_{i j}\right) p^{t-t_{i j}}}{\sum_{i} f_{i j}\left(t_{i j}\right) w_{i}\left(t_{i j}\right) p^{t-t_{i j}}}=\left\langle r_{i j}\right\rangle_{i}
$$

wherein $r_{j}(t)$ is the rating of an online information object, $j$, at time $t, i$ is an index of the rating user, $r_{i j}(t)$ is the rating of the online information object, $j$, given by rating user $i, w_{i}(t)$ is the rating given at time $t, p$ is a decay factor ranging from 0 to 1 , and $f$ is a reliability factor,

wherein the rating of the online information object, $j$, over the plurality of rating users is further weighed by a variance of ratings over the plurality of rating users, and

wherein the rating, $r_{j}(t)=N_{j}(t) / D_{j}(t)$, of the online information object, $j$, over the plurality of rating users is stored in computer memory as a nominator, $N_{j}(t)=N_{j}\left(t^{\prime}\right) p^{t-t^{\prime}}+r_{i j}(t) f_{i j}(t) w_{i}(t)$, and a denominator, $D_{j}(t)$ $=D_{j}\left(t^{\prime}\right) p^{t-t^{\prime}}+f_{i j}(t) w_{i}(t)$, and a previous updating time, $t^{\prime}$.

27. The reputation-based online information filtering system of claim 16, wherein the specified online information object is one or more of an item of content or posting relating to a product, company, or subject.

28. A computer-implemented method, comprising:

instantiating a plurality of system components, implemented using one or more computer systems configured with executable instructions, wherein one or more of the plurality of system components is configured to 
perform sensing, information processing, valuation computation, and/or information exchange;

maintaining a system component data structure, wherein a system component data structure comprises data about a system component;

maintaining an action data structure comprising a plurality of action data records, wherein an action data record comprises data about an action of a system component of the plurality of system components;

maintaining an interaction data structure comprising interaction records, wherein an interaction record is a record of an interaction between two or more system components of the plurality of system components;

determining, for each given system component in the plurality of system components, a valuation of prospective actions and prospective interactions between the given system component and other system components;

determining possible feedback effects;

determining a plurality of possible value exchanges;

determining for the given system components in the plurality of system components, valuations of the prospective actions and the prospective interactions between the given system component and other system components when the possible feedback effects or possible value exchanges are added to the prospective actions and prospective interactions, based at least upon a data analysis of previous interactions or a computer simulation; and identifying, based on computer analyses, whether a condition exists in which proposed interventions result in the prospective interactions being converted into favorable interactions with improved valuations.

29. The computer-implemented method of claim 28, wherein the proposed interventions are used to prevent negative valuations.

30. The computer-implemented method of claim 28, wherein the possible value exchanges or the possible feedback effects are based on distributed computations such that other system components are excluded from access to sensitive data.

31. The computer-implemented method of claim 28, wherein the possible value exchanges and the possible feedback effects further consider expected behaviors of system components based on averages of actually measured behaviors or desired behaviors based on stated preferences or maximum possible values of certain goal functions.

32. The computer-implemented method of claim 28, wherein a multidimensional feedback system and value exchange system is implemented to support self-organization of a system comprising a plurality of interacting system components.

33. The computer-implemented method of claim 28 , wherein the valuations of the prospective actions and the prospective interactions take into account a reputation value of a plurality of reputation values related to system components, actions, or interactions.

34. The computer-implemented method of claim 33, wherein the reputation value is determined by sensor measurements. 
35. The computer-implemented method of claim 33, wherein the reputation value is determined by reputation filters.

36. The computer-implemented method of claim 33, wherein the reputation value is used to define values in a value exchange system.

37. The computer-implemented method of claim 33, wherein the reputation value is determined by ratings.

38. The computer-implemented method of claim 37, wherein ratings of different system components are scaled in such a way that each considered system component has a same overall weight when rating system components, actions, or interactions, independently of a number of ratings sent.

39. The computer-implemented method of claim 37 , wherein a rating of a specified online information object over a plurality of rating system components is determined according to an equation:

$$
r_{j}(t)=\frac{\sum_{i} r_{i j}\left(t_{i j}\right) f_{i j}\left(t_{i j}\right) w_{i}\left(t_{i j}\right) p^{t-t_{i j}}}{\sum_{i} f_{i j}\left(t_{i j}\right) w_{i}\left(t_{i j}\right) p^{t-t_{i j}}}=\left\langle r_{i j}\right\rangle_{i}
$$

wherein $r_{j}(t)$ is the rating of an online information object, $j$, at time $t, i$ is an index of the rating system component, $r_{i j}(t)$ is the rating of the online information object, $j$, given by rating system component $i, w_{i}(t)$ is the rating given at time $t, p$ is a decay factor ranging from 0 to 1 , and $f$ is a reliability factor,

wherein the rating of the online information object, $j$, over the plurality of rating system components is further weighed by a variance of ratings over the plurality of rating system components, and wherein the rating, $r_{j}(t)=N_{j}(t) / D_{j}(t)$, of the online information object, $j$, over the plurality of rating system components is stored in computer memory as a nominator, $N_{j}(t)=N_{j}\left(t^{\prime}\right) p^{t-t \prime}+r_{i j}(t) f_{i j}(t) w_{i}(t)$, and a denominator, $D_{j}(t)=D_{j}\left(t^{\prime}\right) p^{t-t^{\prime}}+f_{i j}(t) w_{i}(t)$, and a previous updating time, $t^{\prime}$

40. A computer-implemented method, comprising:

instantiating a plurality of system components, implemented using one or more computer systems configured with executable instructions, wherein one or more of the plurality of system components is configured to perform sensing, information processing, valuation computation, and/or information exchange;

maintaining a system component data structure, wherein a system component data structure comprises data about a system component; maintaining an action data structure comprising a plurality of action data records, wherein an action data record comprises data about an action of a system component of the plurality of system components;

maintaining an interaction data structure comprising interaction records, wherein an interaction record is a record of an interaction between two or more system components of the plurality of system components; 
evaluating components of a prospective interaction among a set of considered system components, wherein considered system components include references to relative valuations of a set of prospective system components of the prospective interaction;

aligning value changes of a system and at least one component of the prospective interaction according to a respective valuation of interactions or potential interactions;

determining, based on computer analysis of the prospective interaction and a rules data structure that defines value exchanges and feedback effects, whether a prospective value exchange or a prospective feedback effect, when added to the prospective interaction, results in an unfavorable interaction as to a particular system component being converted into a favorable interaction as to that particular system component; and

flagging the prospective interaction as being a favorable interaction, an unfavorable interaction, or a semi-favorable interaction, wherein a semifavorable interaction is defined as an interaction that is convertible into a favorable interaction via a value exchange, wherein an unfavorable interaction is defined as an interaction wherein at least one system component of the set of prospective system components has a negative valuation of the prospective interaction, and a favorable interaction is defined as an interaction wherein each system component of the set of prospective system components has a positive valuation of the prospective interaction.

41. The computer-implemented method of claim 40, wherein the prospective interaction comprises exchange of sensitive data and the sensitive data is managed in a distributed way by third-party computer systems such that excluded from access by other system components.

42. The computer-implemented method of claim 40, considering a reputation value of a plurality of reputation values related to system components, actions, or interactions.

43. The computer-implemented method of claim 42, wherein the reputation value is determined by sensor measurements.

44. The computer-implemented method of claim 42, wherein the reputation value is determined by reputation filters.

45. The computer-implemented method of claim 42, wherein the reputation value is used to define values in a value exchange system.

46. The computer-implemented method of claim 42, wherein the reputation value is determined by ratings.

47. The computer-implemented method of claim 46, wherein ratings of different system components are scaled in such a way that every considered system component has the same overall weight when rating system components, actions, or interactions, independently of a number of ratings sent.

48. The computer-implemented method of claim 46, wherein a rating of a specified online information object over a plurality of rating system components is determined according to an equation: 


$$
r_{j}(t)=\frac{\sum_{i} r_{i j}\left(t_{i j}\right) f_{i j}\left(t_{i j}\right) w_{i}\left(t_{i j}\right) p^{t-t_{i j}}}{\sum_{i} f_{i j}\left(t_{i j}\right) w_{i}\left(t_{i j}\right) p^{t-t_{i j}}}=\left\langle r_{i j}\right\rangle_{i}
$$

wherein $r_{j}(t)$ is the rating of an online information object, $j$, at time $t, i$ is an index of the rating system component, $r_{i j}(t)$ is the rating of the online information object, $j$, given by rating system component $i, w_{i}(t)$ is the rating given at time $t, p$ is a decay factor ranging from 0 to 1 , and $f$ is a reliability factor,

wherein the rating of the online information object, $j$, over the plurality of rating system components is further weighed by a variance of ratings over the plurality of rating system components, and wherein the rating, $r_{j}(t)=N_{j}(t) / D_{j}(t)$, of the online information object, $j$, over the plurality of rating system components is stored in computer memory as a nominator, $N_{j}(t)=N_{j}\left(t^{\prime}\right) p^{t-t \prime}+r_{i j}(t) f_{i j}(t) w_{i}(t)$, and a denominator, $D_{j}(t)=D_{j}\left(t^{\prime}\right) p^{t-t^{\prime}}+f_{i j}(t) w_{i}(t)$, and a previous updating time, $t^{\prime}$.

49. A computer-implemented method for promoting participatory value or information exchange, comprising:

under the control of one or more computer systems configured with executable instructions,

promoting responsible exchange by at least partial transparency of transactions;

representing a plurality of money categories; and

introducing at least one transaction charge for converting among the plurality of money categories.

50. The computer-implemented method of claim 49, wherein the at least one transaction charge is an exchange fee and/or a tax.

51. The computer-implemented method of claim 49, further comprising distinguishing among money of the plurality of money categories in order to encourage particular kinds of consumption or real investments or other desired effects, actions, or interactions.

52. The computer-implemented method of claim 49, wherein the plurality of money categories comprises cash, real electronic money, virtual electronic money, and/or multi-dimensional money.

53. The computer-implemented method of claim 49, wherein the prospective interaction comprises exchange of sensitive data and the sensitive data is managed in a distributed way by third-party computer systems such that excluded from access by other system components.

54. The computer-implemented method of claim 49, considering a reputation value of a plurality of reputation values related to system components, actions, or interactions.

55. The computer-implemented method of claim 54, wherein the reputation value is determined by sensor measurements.

56. The computer-implemented method of claim 54, wherein the reputation value is determined by reputation filters. 
57. The computer-implemented method of claim 54, wherein the reputation value is used to define values in a value exchange system.

58. The computer-implemented method of claim 54, wherein the reputation value is determined by ratings.

59. The computer-implemented method of claim 58, wherein ratings of different system components are scaled in such a way that every considered system component has the same overall weight when rating system components, actions, or interactions, independently of number of ratings sent.

60. The computer-implemented method of claim 58, wherein a rating of a specified online information object over a plurality of rating system components is determined according to an equation:

$$
r_{j}(t)=\frac{\sum_{i} r_{i j}\left(t_{i j}\right) f_{i j}\left(t_{i j}\right) w_{i}\left(t_{i j}\right) p^{t-t_{i j}}}{\sum_{i} f_{i j}\left(t_{i j}\right) w_{i}\left(t_{i j}\right) p^{t-t_{i j}}}=\left\langle r_{i j}\right\rangle_{i}
$$

wherein $r_{j}(t)$ is the rating of an online information object, $j$, at time $t, i$ is an index of the rating system component, $r_{i j}(t)$ is the rating of the online information object, $j$, given by rating system component $i, w_{i}(t)$ is the rating given at time $t, p$ is a decay factor ranging from 0 to 1 , and $f$ is a reliability factor,

wherein the rating of the online information object, $j$, over the plurality of rating system components is further weighed by a variance of ratings over the plurality of rating system components, and

wherein the rating, $r_{j}(t)=N_{j}(t) / D_{j}(t)$, of the online information object, $j$, over the plurality of rating system components is stored in computer memory as a nominator, $N_{j}(t)=N_{j}\left(t^{\prime}\right) p^{t-t \prime}+r_{i j}(t) f_{i j}(t) w_{i}(t)$, and a denominator, $D_{j}(t)=D_{j}\left(t^{\prime}\right) p^{t-t^{\prime}}+f_{i j}(t) w_{i}(t)$, and a previous updating time, $t^{\prime}$.

Open Access This chapter is licensed under the terms of the Creative Commons Attribution 4.0 International License (http://creativecommons.org/licenses/by/4.0/), which permits use, sharing, adaptation, distribution and reproduction in any medium or format, as long as you give appropriate credit to the original author(s) and the source, provide a link to the Creative Commons license and indicate if changes were made.

The images or other third party material in this chapter are included in the chapter's Creative Commons license, unless indicated otherwise in a credit line to the material. If material is not included in the chapter's Creative Commons license and your intended use is not permitted by statutory regulation or exceeds the permitted use, you will need to obtain permission directly from the copyright holder. 